\title{
OppA's deep pockets
}

Bacteria scavenge peptides from their environment through oligopeptide transport (opp) systems. The Lactococcus lactis opp system has the unique property of transporting peptides of a wide range of sizes, which increases the number of peptides that can be taken up. In a recent issue of The EMBO Journal,
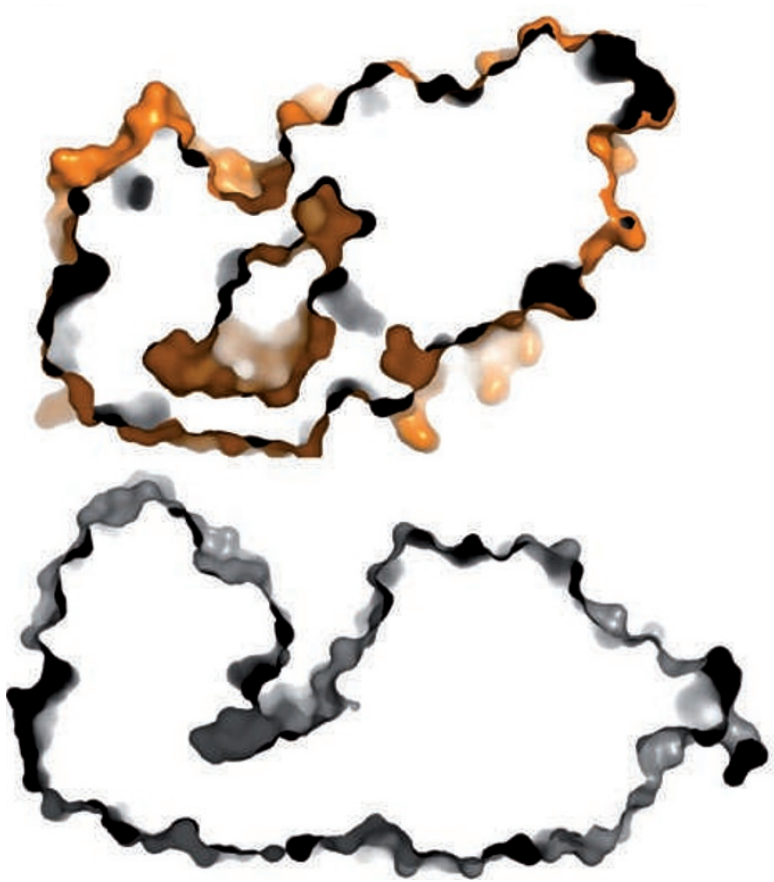

A sliced surface representation of the closed structure (orange) and the open structure (grey) of OppA that highlights the voluminous peptide-binding cavity. Figure courtesy of R.P.-A. Berntsson and D.J. Slotboom, University of Groningen, The Netherlands.
Berntsson and colleagues reveal the crystal structure of OppA and explain this unique property.

Many bacteria use peptides from the environment as a source of amino acids. The opp transporter, a large multiprotein complex located in the bacterial plasma membrane, binds and transports these peptides to the bacterial cytosol. Most bacteria take up short peptides of a narrow size range of several amino acids. By contrast, L. lactis can take up peptides that range in length from 7 to 35 residues. The size range is determined by the OppA protein, which interacts with the peptides through its peptide-binding cavity and then delivers them to the transport machinery. Berntsson and colleagues have determined the crystal structure of OppA from L. lactis bound to a mixture of peptides, and show that OppA has an enlarged ligand-binding cavity. Two loops that in many OppA orthologues confine the binding cavity are moved to the surface of the L. lactis protein, resulting in a peptide-binding cavity that is nearly twice as large as the Bacillus subtilis OppA orthologue, which binds nonapeptides.

Purification and mass spectrometry analysis of the peptides bound to the recombinant OppA revealed that they ranged in size from 7 to 26 residues, although there was a preference for peptides between 9 and 17 residues, and that they were rich in prolines.

Co-crystallization with a specific nonapeptide allowed insight into the interaction of the protein with the peptide. A remarkably hydrophobic protein pocket was noted that accommodates one of the side chains of the peptide. In crystal structures with the mixed peptides, this pocket was often occupied by an isoleucine, which provided a small amount of sequence specificity. Interestingly, L. lactis needs to acquire isoleucine from the environment, so this specificity may increase its ability to acquire this essential nutrient. All other side chains of the peptide were in the aqueous binding cavity, and therefore there were no sequence-specific interactions. The remaining interactions involved the peptide backbone, again explaining the lack of sequence specificity.

L. lactis is often associated with the protein-rich environment of milk, and proteolyses the caseins in milk, which are often rich in prolines. Its ability to take up peptides of a large size range allows it to take full advantage of this environmental pool of amino acids.

Christiaan van Ooij

ORIGINAL RESEARCH PAPER Berntsson, R.

P.-A. et al. The structural basis for peptide selection by the transporter OppA. EMBO J. 19 Mar 2008 (doi: 10.1038/emboj.2009.65) 